\title{
Numerical fatigue life estimation of aluminium 6201-T81 wires containing geometric discontinuities.
}

\author{
Jesús Martínez, Vitor Adriano, José Araújo, Jorge Ferreira, and Cosme da Silva. \\ University of Brasilia, Department of Mechanical Engineering, Brasilia CEP 70910-900, Brazil
}

\begin{abstract}
Previous works have shown that fretting fatigue can be modelled as a notch problem due to the mild superficial damage produced in the partial slip regime during fretting. In this context, the aim of this numerical and experimental study is to estimate the fatigue life of aluminium 6201-T81 wires, used in overhead conductors, containing geometric discontinuities. These discontinuities induce a stress field analogous to the fretting problem. To perform the fatigue life assessments the $L_{M}-N_{f}$ relationship, which is an extension of the Theory of Critical Distance (TCD), was applied. The $L_{M}-N_{f}$ relationship was calibrated using two $S-N$ curves, one of a plain wire and other of a notched wire. The validation of the numerical approach estimations was performed using a wire containing a through hole. The estimations were fallen almost entirely among factor 3 bands when compared with the experimental results. This factor 3 represents the biggest scatter in the $S-N$ curves used to calibrate the $L_{M}-N_{f}$ relation. The TCD approach applied, in terms of point method, provide accurate life estimations in the range $10^{5}$ and $10^{6}$ cycles.
\end{abstract}

\section{Introduction}

Failure in overhead conductor, used in power transmission lines, is mainly associated to the fretting fatigue. Such cables are normally composed of aluminium wires twisted around a core and it is perhaps the most important component in the transmission lines [1-3]. The fatigue failure in these cables are induced by Aeolian vibration and frequently occur inside the "rigid" device where the conductor's motion is constrained, such as suspension clamps, spacers, spacer dampers or Stockbridge dampers. The suspension clamp is one of the most critical devices in terms of conductor fretting fatigue [4]. The Aeolian vibration is caused by the vortices and these are formed alternately from the top and bottom of the conductor. Hence, this vibration causes the cable to bend in the vicinity of a "rigid" device $[5,6]$. The combination of the bending stress, the mean stretching load of the conductor and the clamping force generate complex mechanical interactions due to the relative movements between cable wires and between the cable external wires and the suspension clamp. Some of this interactions causes slip and high multiaxial stress state in the contact zones [4, 7]. For this reason, the failure in the conductor is recognized as a fretting fatigue problem [8].

The fatigue phenomenon appears to be a wellunderstood problem for some structures however this is not the case for overhead conductors. Currently, most of the publications $[1-4,7]$ in fatigue failure of overhead conductor are oriented to assessment the nominal stress, related to the bending movement of the conductor, and the mean stretching loads using the Poffenberger-Swart (P-S) formula [9]. However, the P-S formula is semiempirical and provides nominal idealized stress which is used to establish and compare the fatigue strength of different conductors based on bending stress. Nevertheless, this approach cannot allow describing the fretting fatigue phenomenon in the failure regions. The numerical simulation tools are solutions to obtain the state of the stress in this specific region where the accessibility is quite difficult. However, the numerical simulation could be quite complex and very expensive in some cases [10].

On the other hand, some works [11-13] have shown that the fretting fatigue can be modelled as a notch problem, due to the fact that in both cases the fatigue exist, a multiaxial stress state and high stress gradients. In this setting, this approach allows the use of different methods to estimate the fatigue life, normally used to assessment the notched components, in some fretting fatigue cases.

The study of the notched components is quite important in mechanical engineer studies. Early work to estimate the fatigue strength were based on the concentration factor $\left(k_{t}\right)$. This estimation can be correct for high-cycle fatigue strength of blunt notches, but not for sharp notches, for which the estimations are too conservative [14]. For a better representation of fatigue strength reduction at long lives, the fatigue notch factor

\footnotetext{
Corresponding author: author@e-mail.org
} 
$\left(k_{f}\right)$ was introduced to the analyses. This factor is the relation between plain and notched specimen fatigue strength and is formally defined only for completely reversed stresses [15]. The discrepancy between $k_{f}$ and $k_{t}$ can be quantified by the "notch sensitivity" $(q)$. This factor is used to describe the effect of a notch on the fatigue strength of the specimen in linear elastic analyses. In this setting, Neuber [16] and Peterson [17] proposed the notch-sensitivity formulas in terms of a "empirical material constant". They argue that the stresses around the notch should be averaged to obtain an effective stress $\left(\sigma_{\text {eff }}\right)$ that could be linked to the fatigue damage process zone. Latter, the "material constant" was formalized in a unifying theoretical model by Taylor [18] in four methods to evaluate the equivalent stress. The group of this methods is known as the Theory of Critical Distance (TCD) that have shown high accurate results to predict the fatigue limit of notched components $[19,20]$.

The TCD is a nonlocal approach to predict the fatigue limit of notched component by using the stress field around the notch. This theory was extended by Taylor [18] who applied the same theory to the components with other geometrical discontinuities. In addition, Susmel and Taylor [14] reviewed the critical distance concepts and extended the TCD to perform fatigue life assessments in the medium cycle regime based on the assumption that the critical distance $\left(L_{M}\right)$ changes with the number of cycles to failure $\left(N_{f}\right)$ : the $L_{M}$ $-N_{f}$ relationship. This idea, allow to estimate the fatigue strength/life, in the same load ratio, and not just the fatigue limit like an early TCD approach.

The $L_{M}-N_{f}$ relationship was used in this study to perform the fatigue life estimation of aluminium wires containing geometric discontinuities. A sharp notch and a through hole. The aim of this experimental and numerical work is to evaluate this approach in the aluminium 6201-T81 wire. For this reason, it was necessary to establish the methodology for an experimental campaign to obtain the $S-N$ curves and calibrate $L_{M}-N_{f}$ relationship. The point method (PM) of the TDC was used for the calibration stage and for the fatigue life estimation. This is a preliminary study in single wires, which could contribute to analyses of fretting fatigue behaviour and life fatigue estimation on overhead conductor cables.

\section{$2 L_{M}-N_{f}$ Relationship}

The Theory of Critical Distance (TCD) is a group of methods to assess the effective stress in a specific distance. Experimental results shown $[18,21]$ that the critical distance values, for any method, depends of the material characteristic length $(L)$ [19]:

$$
L=\frac{1}{\pi}\left(\frac{\Delta K_{t h}}{\Delta \sigma_{0}}\right)^{2}
$$

where $\Delta \sigma_{0}$ is the plain fatigue limit and $K_{t h}$ is the threshold value of the stress intensity factor. The two parameters, $\Delta \sigma_{0}$ and $K_{t h}$, are determined at the same load ratio, $R$. The main objective of the critical distance theory is to estimate the effective stress which exceeds the plain fatigue limit in the linear-elastic stress distribution around the notch (Fig. 1). Then, this approach assess a region and not the hot spot of notch. This region is known like fatigue process zone. Therefore, the effective stress can be estimated from analyses of the maximum principal stress $\left(\sigma_{1}\right)$ distribution around the geometric discontinuity by four methods: (i) Point Method (PM) at certain distance from the hot-spot of the stress concentrator; (ii) Line Method (LM) from the averaged over a line; (iii) Area Method (AM) from averaged over an area, and (iv) Volume Method (MV) from the averaged over a volume [14, 18, 19].

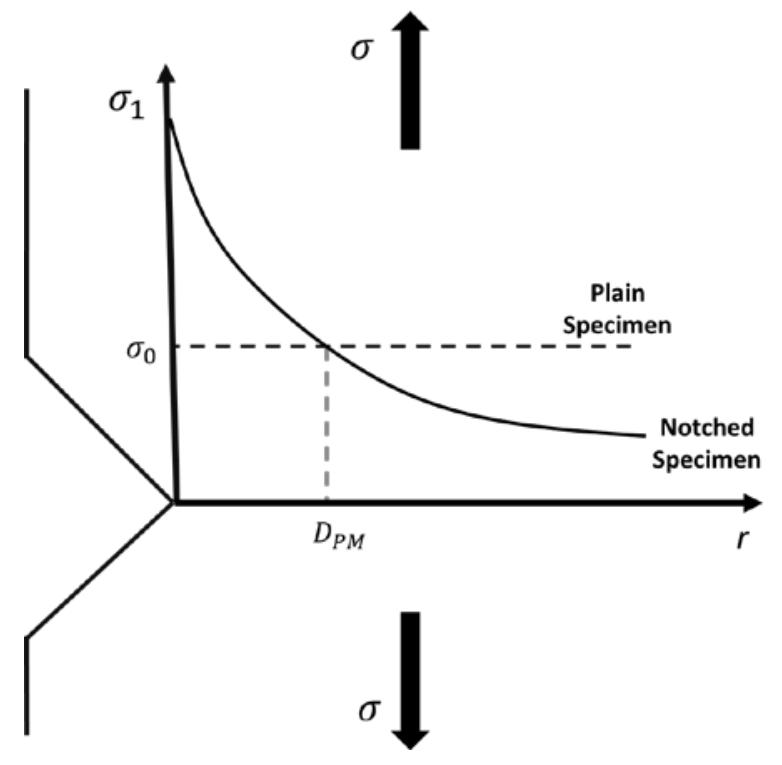

Fig. 1. Linear-elastic stress distribution and effective stress calculated according to the Point Method.

The methods of the TDC are based in the linear elastic mechanics concepts and experimental results which define the critical distance $[18,19]$. According to the above definitions, the PM (2), LM (3), AM (4) and MV (5) can be formalized in the fallowing expressions:

$$
\begin{gathered}
\Delta \sigma_{e f f}=\Delta \sigma_{1}(r)=\Delta \sigma_{0} \\
\Delta \sigma_{e f f}=\frac{1}{l} \int \Delta \sigma_{1}(r) d r=\Delta \sigma_{0} \\
\Delta \sigma_{e f f}=\frac{1}{A} \int \Delta \sigma_{1}(\theta, r) d A=\Delta \sigma_{0} \\
\Delta \sigma_{e f f}=\frac{1}{V} \int \Delta \sigma_{1}(\varnothing, \theta, r) d V=\Delta \sigma_{0}
\end{gathered}
$$

These expressions are used to predict the fatigue limits in notched specimens $[19,20]$. One of the applications of this theory is to estimate lifetime of notch components in the medium-cycle fatigue regime proposed by Susmel and Taylor [14]. They proposed that the material characteristic length changes with the number of the cycles to failure $\left(N_{f}\right)$. Then, the material characteristic determined in the medium-cycle fatigue regime $\left(L_{M}\right)$ is defined as: 


$$
L_{M}\left(N_{f}\right)=A\left(N_{f}\right)^{B}
$$

where $A$ and $B$ are material constants that result of the calibration process. These material constants are expected to be different for dissimilar materials and varying load ratios, $R$. Also, Susmel and Taylor [14] show that the better strategy to calibrate the $L_{M}-N_{f}$ relation is using two fatigue curves ( $S-N$ curves), one for a plain specimens and the other for the notch specimens, in the same load ratio. The same strategy used in this work and the calibration process are explained in the following sections.

\section{Methodology}

This section describes the experimental details and the numerical simulations used to estimate the fatigue life of the wires containing geometrical discontinuities.

\subsection{Experimental procedure}

The test specimens are wires taken from the aluminium conductor cable used in the overhead transmission lines. These wires were machined to transform in test specimens of the three different geometries. The dimensions of these specimens are shown in Fig. 2.

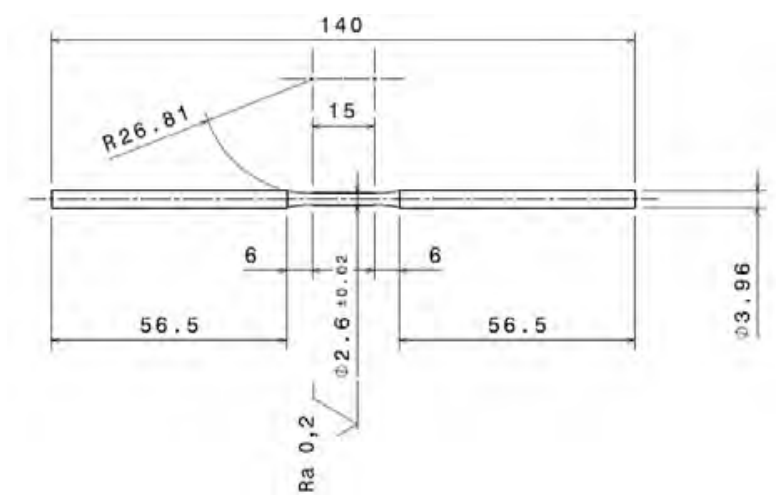

(a)

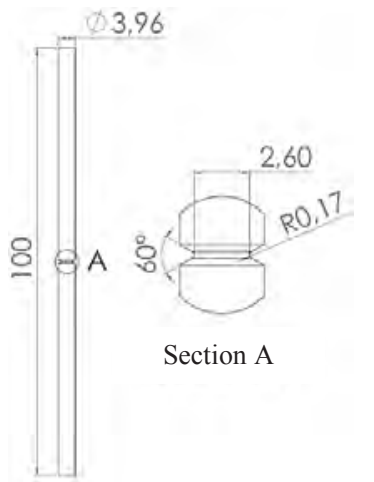

(b)

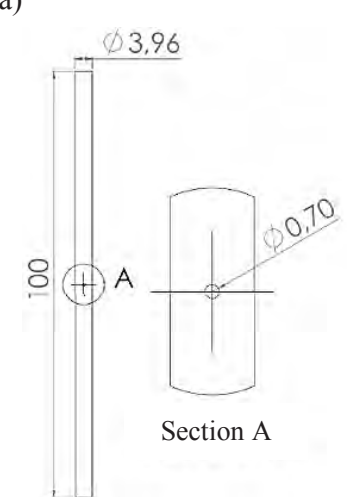

(c)
Fig. 2. Geometries of the test specimens (a) Plain wire, (b) Notch wire and (c) Wire containing a through hole.

The plain specimen was machined with special careful until a superficial roughness $\leq 0.2 \mathrm{Ra}$ was reached. The roughness measurements were performed by a Confocal Laser Microscopy Olympus LEXT
OLS4100 in different regions of the reduced crosssection. Also, the geometrical characteristics for the sharp notched wires $\rho=0.17$ were verified.

All fatigue tests were load controlled and conducted in a stress ratio $R=-1$ with frequencies varying from 10 to $40 \mathrm{~Hz}$ in the servohydraulic machine MTS. The fatigue test were conducted until the failure or the specimens were considered "run-out" if they survived 5 x $10^{6}$ cycles.

\subsection{Finite element analysis (FEA)}

The TDC is based on the determination of the stress field into the material in the stress concentration region or process zone. To determine the stress field the use of numerical analyses like a finite element method is a powerful tool. However, this method has some limitations with regard to the size and complexity of components that can be modelled [19]. Furthermore, the computational cost is another topic to be considered. In this study, this stress field around the notched wire and the wire containing a through hole were obtained by using the commercial finite element software Abaqus. Therefore, these geometries were designed in the simplified three dimensional model with $1 / 4$ of the specimen (Fig 3). In the initial model were established symmetry conditions and a low mesh density with tennode tetrahedral elements (C3D10). Afterward, it was created a submodel around the geometrical discontinuities with a very fine mesh with eight-node hexahedral elements (C3D8R). Converge analyses helped to establish the mesh refinement. These strategies allowed to obtain good results of the stress field around the geometrical discontinuity in a reasonable computes times.

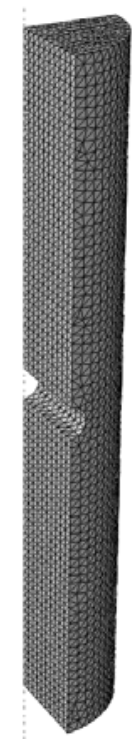

(a)

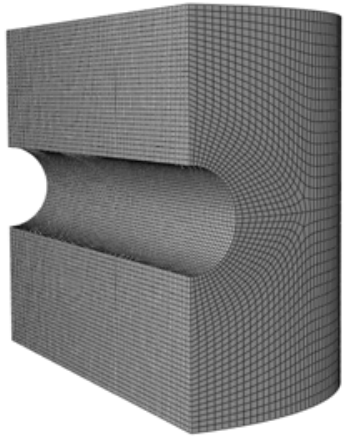

(b)
Fig. 3. The simplified three dimensional model of the wire containing a through hole, (a) initial model and (b) submodel. 


\subsection{Calibration of the $L_{M}-N_{f}$ relationship}

The strategy used to calibrate the $L_{M}-N_{f}$ relationship in this work was presented by Susmel and Taylor [14]. This calibration was performed by using two fatigue curves, one for a plain wire and the other for the notch wire, for the same load ratio. In the other hand, to determine the stress field, it was necessary to create a threedimensional model of the sharp notch and thereafter to perform the FEA. This analyse gives the stress distribution in the region of the stress concentrator, as shown in Fig. 1. At the end, it was necessary to establish the critical distance for the both specimen, plain and notched, which present approximately the same fatigue life. This process is repeated several times to find the enough points to fit a $L_{M}-N_{f}$ relationship. The Fig. 4 presents the flowchart which summarizes the calibration process described above.

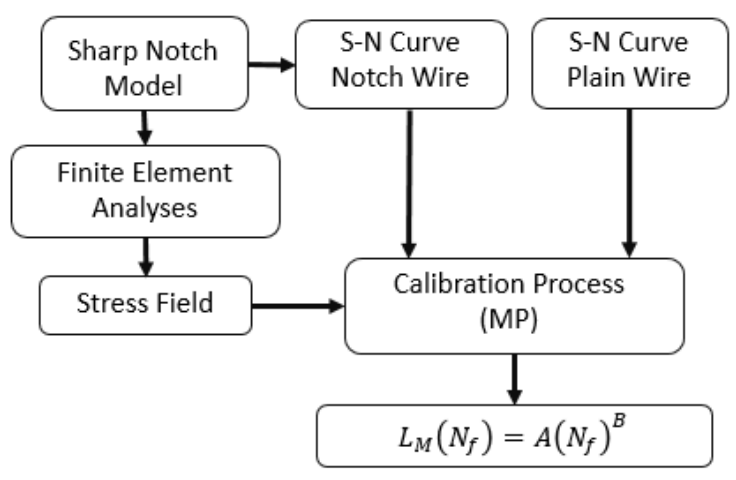

Fig. 4. Flowchart summarizing the calibration process.

\subsection{Procedure to estimate the fatigue Life}

The life estimation was performed by using an iterative process in the numerical algorithm. This algorithm was developed in a Matlab code to compare two fatigue lives: an initial arbitrary value and a material fatigue life obtained from the $S$ - $N$ curve of the plain wire. The initial arbitrary fatigue life was used to obtain the critical distance. From this critical distance, the maximum principal stress $\left(\sigma_{1}\right)$ is determinated from the linearelastic distribution around the geometric discontinuity. The calculated value of $\sigma_{1}$ allows to estimate the "first failure life" from the $S-N$ relation of the plain wire. Now, the two fatigue lives are compared. The iterative process was stopped when the both fatigue lives are approximately the same (tolerance $<5 \%$ ). If not, the arbitrary fatigue life is incremented and the procedure is repeated until the code has reached its convergence [14].

\section{Results}

This section presents the results of fatigue test for the plain wire, notch wire and wire containing a through hole with diameter of $0.7 \mathrm{~mm}$. The $L_{M}-N_{f}$ relationship in terms of the PM and the fatigue life estimation are also presented.

\subsection{Fatigue tests}

The fatigue tests results are show in log-log graph (Fig. 5) and the amplitude stress levels correspond to the nominal gross stress. The $S-N$ curves for the plain wire, notch wire and wire containing a through hole are in the same graph aiming the evaluation of notch effect at fatigue lives of aluminium wires. Also, in the graph are presented the $95 \%$ confidence bands. Each point in the graph (Fig. 5) represents the fatigue life and the points with arrow represent the "run-out" tests.

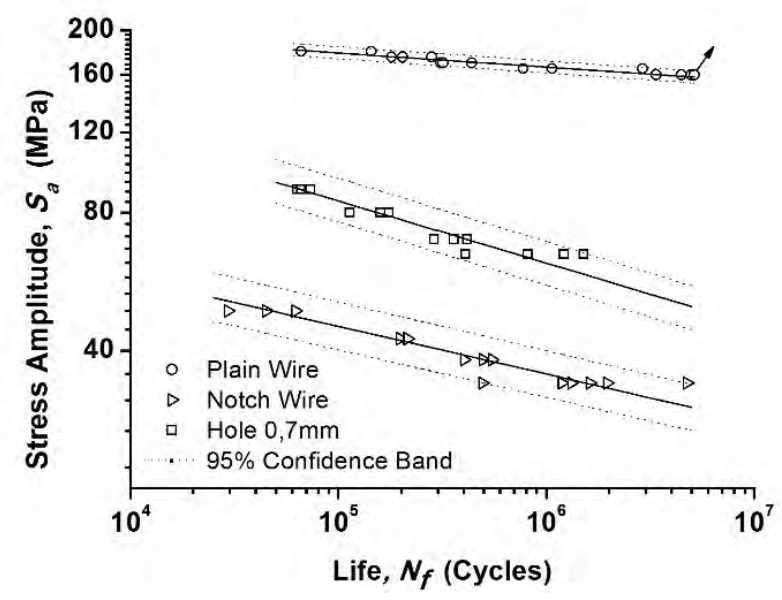

Fig. 5. $S-N$ Curves of the plain wire, notch wire and wire containing a through hole $0.7 \mathrm{~mm}$ of diameter.

\section{2 $L_{M}-N_{f}$ Relationship by point method}

From results of the calibration procedure made using the two S-N curves, the $L_{M}-N_{f}$ relationship was established in terms of the PM:

$$
L_{M}\left(N_{f}\right)=0,6388\left(N_{f}\right)^{-0,1963}
$$

where the constants from the calibration procedure can only be used for this specific material and for the fully reverse uniaxial loadings. The above equation was determined using $L_{M}$ values calculated between $3 \times 10^{4}$ and $5 \times 10^{6}$ cycles to failure, approximately. This relationship was used in the algorithm to determine the critical distance in terms of the PM and in consequence, the fatigue life for the medium-cycle regime.

\subsection{Determination of the fatigue life estimation}

The results of the fatigue life estimation for the mediumcycle regime are show in Fig. 6. This graph compares the experimental life $\left(N_{f}\right)$ and the estimated life $\left(N_{e}\right)$. The straight line represents the perfect fatigue life estimation. Region above this line is considered a conservative life estimation and below of the line is a non conservative region. Furthermore, the dashed lines represent the factor 3 bands as the admissible limit error for the estimated life. The factor 3 was select because it represents the biggest scatter of the experimental fatigue data in the $S$ $N$ curves. 


\section{Discussion}

The fatigue test results shown that aluminium alloy 6201-T81 is extremely sensitive to the fatigue phenomenon. In the $S-N$ curves, little changes (5 MPa) in the stress amplitude causes change of one order of magnitude in the fatigue life. This proves that the estimation of the fatigue life is a better approach to describe the aluminuim 6201-T81 fatigue behavior rather than use the fatigue strength.

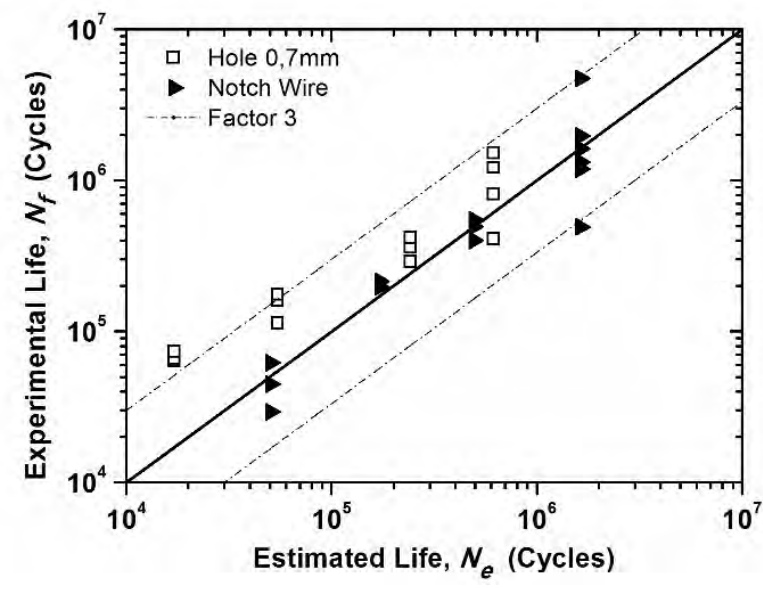

Fig. 6. Fatigue life estimation for the notch wire and wire containing a through hole $0.7 \mathrm{~mm}$.

The chosen parameter to estimate the fatigue damage is the maximum principal stress. This choice is in accordance with the most modern methods to predict the uniaxial high-cycle fatigue strength of notched components [16-18, 22, 23]. Moreover, this idea is supported by the fact that the stage II of the propagation failure is assumed to be predominant over other failure mechanisms. This means that the fatigue damage occurs at the plane perpendicular to the loading direction (maximum loading), so that, the fracture is characterized to be a Mode I from the linear-elastic mechanics. This fact was observed in some test specimens. All wires containing the through hole of $0.7 \mathrm{~mm}$ failed in the plane perpendicular to the loading direction.

The fatigue life estimation for the wire containing a through hole present a good correlation with the experimental results. The majority of data points fall within the scatter band. However, it was observed that the fatigue lives below $10^{5}$ cycles had poor estimations, falling in the conservative region. This result is probably associated with the fact that the experimental data of the through hole have smaller fatigue lives than the fatigue lives of the plain wire in the calibration procedure. It is possible to appreciate a trend in the fatigue life estimation that is conservative for the lives below $10^{5}$ cycles and not conservatives for the lives above $10^{6}$ cycles. In general, the accuracy for the life estimation is between $10^{5}$ and $10^{6}$ cycles.

The accuracy of $L_{M}-N_{f}$ relationship approach to estimate the fatigue life was proved by Susmel and Taylor [14]. However, their results are not enough to establish that this approach always works in different materials, geometric discontinuities and specimen's types. The study of 6201-T81, plain wire and those containing geometric discontinuities, as depicted in the present work offers new challenges which are the geometric and dimensional features. According to this observation, it is important to validate this life estimation approach and to establish some strategies that will be useful to study geometric discontinuities with complex shape. This is the same reason that motivates the use a three-dimensional model for the sharp notch and the through hole in the FEA.

The three-dimensional model is indispensable for the geometric discontinuities with complex shape because of the absence of the symmetry conditions. In this case, the trajectory to describe the stress field in the geometries shapes, such as a notch, is an intuitive process. However, to establish this trajectory for the through hole in the cylindrical specimen is not a simple task. Therefore, the use of the FEA is necessary. To get experience, knowledge and create news strategies are steps to study geometric discontinuities with complex shape. The intermediate steps are necessary because when the complexity of the phenomenon increases, the numerical simulations can be a high demand of computer resources, with expensive costs and unsatisfactory results.

The authors would like to acknowledge the financial support of TAESA by means of the R\&D program of ANEEL. The support of Finatec, CAPES and CNPq are also acknowledged.

\section{Conclusions}

The present work is a numerical and experimental study to estimate the fatigue life of aluminium alloy 6201-T81 wires containing geometric discontinuities. The present work is part of the studies that try to find news methodologies to the fatigue life estimation in the system cable-suspension clamp electric conductors. The main conclusions are summarized as follows:

- The $L_{M}-N_{f}$ relationship, when calibrated with fatigue data from the plain wire and the notched wires, generated good life estimations for the wires containing through hole of $0.7 \mathrm{~mm}$ of diameter. In terms of the PM, the majority of the estimations were located between factor 3 bands and these results were considered satisfactory for the aim of this work.

- The PM need a path to define the stress distribution. In this sense, the strategy to chose the trajectory, for a three-dimensional model of the through hole, was considered successful and gave accurate life estimations between $10^{5}$ and $10^{6}$ cycles.

- The $L_{M}-N_{f}$ relationship and some strategies developed in this study for the AA6201-T81 wires can be used to perform futures wires fretting fatigue analysis based on the TCD. Therefore, this study not only validates the use of the TCD to predict fatigue life in the above mentioned aluminium alloy but also contributes to further fretting fatigue analyses that would improve the life estimation of overhead conductors. 


\section{References}

1. Z. R. Zhou, A. Cardou, M. Fiset, and S. Goudreau, Wear, 173, 179-188 (1994).

2. R.B. Kalombo, J.M.G. Martínez, J.L.A. Ferreira, C.R.M. Da Silva, and J.A. Araújo, Procedia Eng., 133, 223-232 (2015).

3. C.R.F. Azevedo, A.M.D. Henriques, A.R. Pulino Filho, J.L.A. Ferreira, and J.A. Araújo, Eng. Fail. Anal., 16, 136-151 (2009).

4. R. B. Kalombo, M. S. Pestana, J. L. A. Ferreira, C. R. M. da Silva, and J. A. Araújo, Tribol. Int., 108, 141-149 (2017).

5. CIGRÉ-SCB2-WG11-TF7-06-01 (2006).

6. D. Committee, I. Power, and E. Society, IEEE Guide for Aeolian Vibration Field Measurements of Overhead (2007).

7. A.A. Fadel, D. Rosa, L.B. Murça, J.L.A. Fereira, and J.A. Araújo, Int. J. Fatigue, 42, 24-34 (2012).

8. Z.R. Zhou, A. Cardou, S. Goudreau, and M. Fiset, "Fundamental investigations of electrical conductor fretting fatigue," Tribol. Int., vol. 29, no. 3, pp. 221232, (1996).

9. J.C. Poffenberger and R.L. Swart, IEEE Trans. Power Appar. Syst., 84, 508-513 (1965).

10. F. Lévesque, S. Goudreau, L. Cloutier, and A. Cardou, Tribol. Int., 44, 1014-1023 (2011).

11. A.E. Giannakopoulos, T.C. Lindley, and S. Suresh, Acta Mater., 46, 2955-2968 (1998).

12. J.A. Araújo, L. Susmel, D. Taylor, J.C.T. Ferro, and E.N. Mamiya, Int. J. Fatigue, 29, 95-107 (2007).

13. J.A. Araújo, F.C. Castro, S. Pommier, J. Bellacave, and J. Mériaux, 33, 427-433 (2015).

14. L. Susmel and D. Taylor, Fatigue Fract. Eng. Mater. Struct., 30, 567-581 (2007).

15. N.E. Dowling, Mechanical Behavior of Materials: Engineering Methods for Deformation, Fracture, and Fatigue, 4th ed. Pearson Education (2013).

16. H. Neuber, Theory of notch stresses: principles for exact calculation of strength with reference to structural form and material, 2nd ed. U.S. Atomic Energy Commission, Office of Tech. Inf. (1958).

17. R. Peterson, Notch Sensitivity, McGraw Hill, 293306 (1959).

18. D. Taylor, Int. J. Fatigue, 21, 413-420 (1999).

19. D. Taylor, The Theory of Critical Distances: A New Perspective in Fracture Mechanics, Elseiver Science Ltda, (2007).

20. L. Susmel, Eng. Fract. Mech., 75, 1706-1724 (2008).

21. K. Tanaka, Y. Nakai, and M. Yamashita, Int. J. Fract., 17, 519-533 (1981).

22. E. I. Haddad, K. N. Smith, and T. H. Topper, J. Eng. Mater. Technol., 101, 42-46 (1979).

23. D. Taylor and G. Wang, Fatigue Fract. Eng. Mater. Struct., 23, 387-394 (2000). 